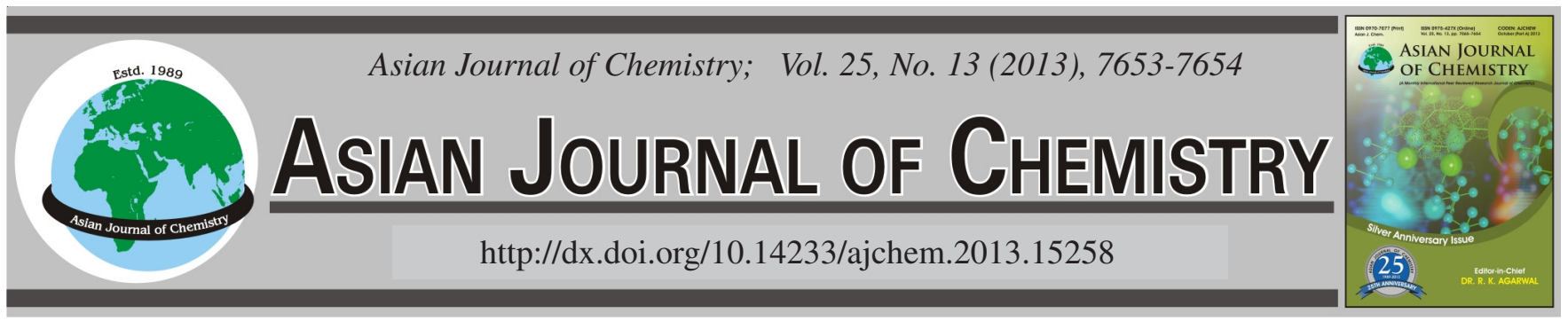

NOTE

\title{
Analysis on Dielectric Properties of Chemical Solution with Potassium Ion Under Different Concentrations
}

\author{
LeI Feng ${ }^{1}$, Jun SHU ${ }^{2}$ and XIAO-QING YANG ${ }^{2, *}$
}

${ }^{1}$ China Academy of Engineering Physics, Mianyang, Sichuan, 621900, P.R. China

${ }^{2}$ College of Electronics and Information Engineering, Sichuan University, Chengdu 610065, P.R. China

*Corresponding author: E-mail: yyxxqq_mail@163.com; sdill2005@126.com

(Received: 31 January 2013;

Accepted: 5 July 2013)

AJC-13780

The dielectric properties of electrolyte solution with $\mathrm{K}^{+}$are studied. In this paper, the changes of complex permittivity of the potassium
nitrate and potassium acetate with different concentrations are measured using coaxial line probe. It is shown that the changes of real part
of the complex permittivity are all small and the changes of imaginary part of the complex permittivity of electrolyte solution are quite
obvious. By analyzing the systems and variation error with different concentrations, a conclusion is drawn that there is an agreement for
reference and measurement results which is important for environmental monitoring, remote sensing detection and imaging.
Key Words: Permittivity, Electrolyte solution, Remote sensing.

The electromagnetic spectrum with different wavelength bands has applications in diverse areas. According to operating wavelength band, ultraviolet remote sensing, visual spectrum remote sensing, infrared remote sensing, microwave remote sensing and multispectral remote sensing are included in remote sensing system. In this paper, the dielectric measurement of chemical solution is under the microwave sensing frequency range.

Dielectric measurements such as dielectric constant, dielectric loss and relaxation time reveal significant information about the chemical and physical characteristics of solution. These properties are drastically affected by the presence of a dopant in the polymer ${ }^{1-5}$. The study of dielectric relaxation provides valuable information about intermolecular interaction and hence miscibility.

In microwave remote sensing, it is important to determine the dielectric properties of study sample. The soil permittivity is a function of soil moisture and soil types ${ }^{6}$. Sea ice is a mixture of ice and saline water and the permittivity determination is a critical issue ${ }^{7}$. The permittivities of dry and wet snow continue to draw interest ${ }^{8}$. In geophysical exploration, the permittivity of fluid filled porous rocks has to be studied ${ }^{9}$. The permittivity measurement becomes more and more important for remote sensing, especially in electrolyte solution.

Potassium ion is distributed widely in electrolyte solution and is one of main pollutant in ground water, it is significant to study the characteristics of electrolyte solution with $\mathrm{K}^{+}$. In this paper, potassium nitrate and potassium acetate are chosen for study.

In this paper, an effective measurement method and thinking is proposed, the real part and the imaginary part of two different electrolyte solutions with $\mathrm{K}^{+}$are measured under different concentrations.

Dielectric permittivity is a frequency-dependent parameter that describes the ability of the medium to polarize in response to an external electrical field.

The experimental system is showed in Fig. 1. The reflection coefficients are obtained from a specially designed coaxial line probe with an Agilent E8362B microwave vector network analyzer. Meanwhile, KXS and a trough are employed to control the temperature of the solution in the beaker and the UMI-8 optical fiber thermometer is measured the temperature of the electrolyte solution.

Table- 1 shows the measurement complex permittivity values of $\mathrm{K}^{+}$electrolyte solution with potassium nitrate and potassium acetate respectively change with different concentrations at $34^{\circ} \mathrm{C}$ and $2.45 \mathrm{GHz}$. Fig. 2 shows the measurement value and reference value ${ }^{10}$ of the real part and imaginary part of potassium nitrate permittivity. Fig. 3 shows the measurement value and reference value of the real part and imaginary part of potassium acetate permittivity.

From Table-1 and Figs. 2 and 3, the variations of real part and imaginary part of complex permittivity values of $\mathrm{K}^{+}$ 


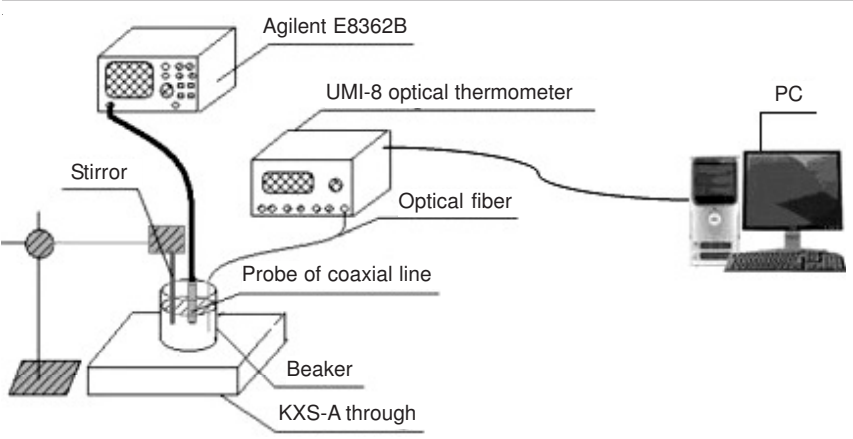

Fig. 1. The experimental system

\begin{tabular}{ccc|cc}
\multicolumn{5}{c}{ TABLE-1 } \\
\multicolumn{4}{c}{$\begin{array}{c}\text { MEASUREMENT COMPLEX PERMITTIVITY } \\
\text { VALUES ON K }\end{array}$ ELECTROLYTE SOLUTION } \\
\hline $\begin{array}{c}\text { Concentration } \\
(\mathrm{mol} / \mathrm{L})\end{array}$ & \multicolumn{2}{c}{ Potassium nitrate } & \multicolumn{2}{c}{ Potassium acetate } \\
\cline { 2 - 5 } $\mathrm{e}_{\text {eff }}^{\prime}$ & $\mathrm{e}_{\text {eff }}^{\prime}$ & $\mathrm{e}_{\text {eff }}^{\prime}$ & $\mathrm{e}_{\text {eff }}$ \\
\hline 0.05 & 83.9364 & 7.8172 & 81.7265 & 19.1101 \\
0.10 & 82.7387 & 9.8155 & 80.8368 & 22.8962 \\
0.15 & 81.5523 & 11.7901 & 79.9782 & 26.5474 \\
0.20 & 80.3757 & 13.7458 & 79.1415 & 30.1109 \\
0.25 & 79.1958 & 15.6854 & 78.3127 & 33.7546 \\
0.30 & 78.0365 & 17.2124 & 77.5024 & 37.3562 \\
0.35 & 76.8421 & 19.5466 & 76.6962 & 40.9824 \\
0.40 & 75.6232 & 21.1553 & 75.8723 & 44.5321 \\
\hline
\end{tabular}

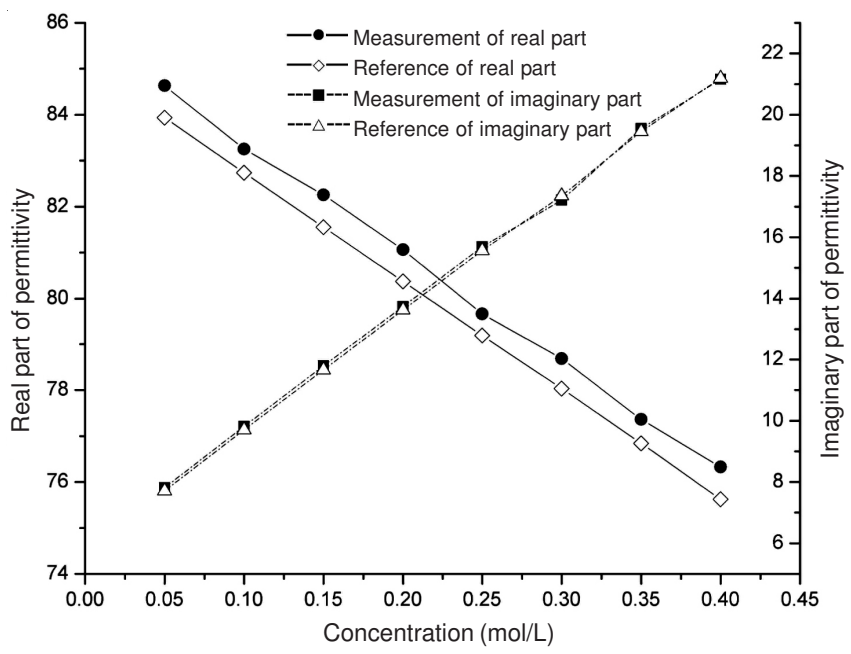

Fig. 2. Real part and imaginary part of potassium nitrate permittivity

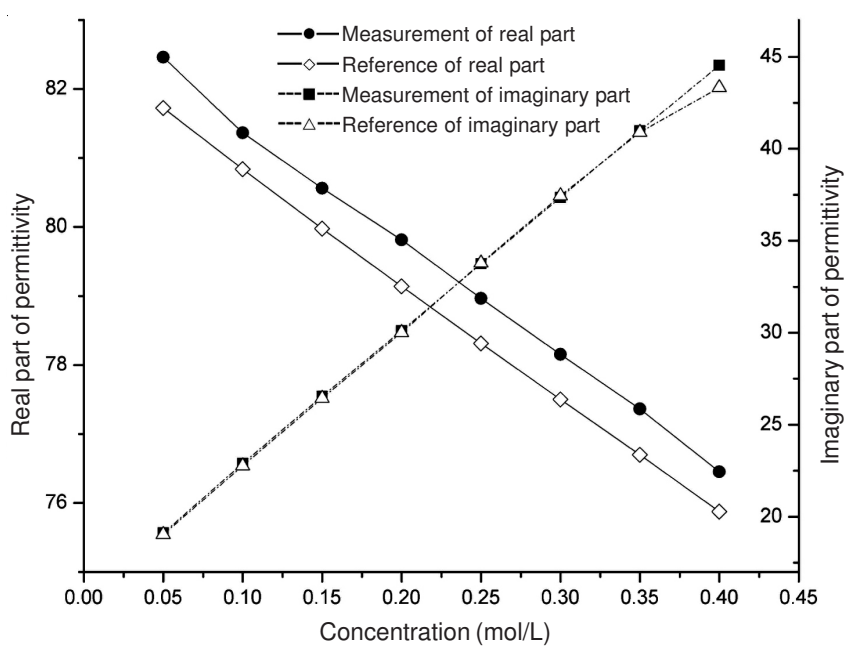

Fig. 3. Real part and imaginary part of potassium acetate permittivity electrolyte solution changes with different concentrations are analyzed as follows.

In Figs. 2 and 3, the real part of potassium nitrate and potassium acetate permittivity are respectively reduced by a little with the increase of concentrations. The maximum relative variations are only 9.9 and $7.16 \%$ from 0.05 to 0.4 $\mathrm{mol} / \mathrm{L}$ concentration. But it is observed that the changes of imaginary part are obvious. The maximum relative variations are set at 170.63 and $133.03 \%$ respectively. From Figs. 2 and 3 it is observed that the measured permittivity reasonably agrees with the reference results.

There is a large acceleration in the course of the imaginary part variation when the concentration is changed, whether the potassium nitrate or potassium acetate electrolyte solution. It may be related to the molecular structure and intermolecular forces of electrolyte solution.

\section{Conclusion}

In this paper it is found that the real part and imaginary part of $\mathrm{K}^{+}$electrolyte solution permittivity changes with different concentrations. Under the band of microwave remote sensing, it is quite obvious that the imaginary part of complex permittivity of $\mathrm{K}^{+}$electrolyte solution changes with the increase of solution concentration. In addition, based on electrolyte solution permittivity research under the microwave frequency, it is known the changes of reflection and absorption of microwaves by the reactants.

Besides, it is helpful to understand the interactions between microwave and solution and provides the study basis for microwave heating. The measured permittivity is important for environmental monitoring, remote sensing detection and imaging.

\section{ACKNOWLEDGEMENTS}

This project was supported by the National Science Foundation of China (No. 61001019)and 973 plan (No. 2013CB328905).

\section{REFERENCES}

1. H. Saito and B. Stuhn, Polymer, 3, 475 (1994).

2. Ch. Ramu, Y.R.V. Naidu and A.K. Sharma, Ferroelectrics, 159, 275 (1994).

3. M.M. Mosad, J. Mater. Sci. Lett., 9, 32 (1990).

4. R. Turch, Phys. State Solids, 121, 119 (1990).

5. Y. Tsujita, Y. Murafuji, K. Yano and A. Takizawa, J. Macromol. Sci. Phys. B, 23, 311 (1984).

6. J.R. Wang and T.J. Schmugge, IEEE Trans. Geosci. Remote Sens., 18, 288 (1980).

7. K.M. Golden, in ed.: G. Papanicolaou, The Interaction of Microwave with Sea Ice, Wave Propagation in Complex Media, IMA Volumes in Mathematics and Its Applications, SpringerVerlag, Berlin, Germany, Vol. 96, pp. 75-94 (1997).

8. W. Linlor, J. Appl. Phys., 23, 2811 (1980).

9. W.C. Chew, J.A. Friedrich and R. Geiger, IEEE Trans. Geosci. Rem. Sens., 28, 207 (1990)

10. X.Q. Yang and K.M. Huang, Acta Electron. Sin., 34, 356 (2006). 